\title{
MDM2 Expression in the Progression of Barrett's Oesophagus
}

Leora Robinson, Mohammad llyas, Krish Ragunath, Tim Card, Berice Abradu-Berchie, Philip Kaye

Nottingham University Hospitals and University of Nottingham, Nottingham Digestive Diseases Biomedical Research Centre

Corresponding Author: Philip Kaye

philip.kaye@nuh.nhs.uk

Dept Histopathology, Queens Medical Centre, Nottingham NG7 2UH 07806792155

No conflicts of interest for any author

Keywords: Barret's oesophagus, MDM2, p53, dysplasia

Word Count: 828 
Sir: Barrett's oesophagus (BO) is the main precursor for oesophageal adenocarcinoma $(O A C)$, and dysplasia arising within it is the standard marker used to predict risk of malignant progression. However, dysplasia diagnosis is subject to a significant amount of interobserver variation. Biomarkers may aid the diagnosis of dysplasia and may help predict the risk of progression to OAC. Abnormal expression of $\mathrm{p} 53$ can be reproducibly detected by immunohistochemistry $(\mathrm{IHC})$ and it is an effective marker of risk of OAC ${ }^{1}$. There is however still a need for additional early stage markers.

MDM2 protein is a negative regulator of $\mathrm{p} 53$ which binds, ubiquitinates and inactivates it. However, MDM2 expression, particularly its relationship to p53 expression and mutation, in tumours appears to be variable and is little studied in $\mathrm{BO}$ and $\mathrm{OAC}^{2}$.

We aimed to assess the expression of MDM2 as a potential marker of neoplastic progression in $\mathrm{BO}$ and investigate its relationships with $\mathrm{p} 53$ expression.

A total of 105 cases of BO were selected from the Barrett's dysplasia database at NUH from 2003 to 2016. Selected cases included a sample of ND, ID that progressed or did not progress to dysplasia or cancer as well as a sample of cases with LGD, HGD and OAC. Twelve cases were excluded due to lack of representative tissue. The remaining 93 cases were tested by IHC for MDM2 expression in formalin-fixed paraffin-embedded tissue samples. IHC was performed using MDM2 antibody (clone: ab3110, Abcam, Cambridge, UK) following citrate buffer antigen retrieval, at 1:2000 dilution in Leica antibody diluent (AR9352; Leica, Milton Keynes, UK). Samples were stained for p53 with D07 antibody (Dako, Ely, UK) using the Roche Ventana Ultra Benchmark system with automated heat mediated epitope retrieval. MDM2 and p53 immunostaining was assessed with H\&E for each case. P53 staining was scored using a qualitiative approach based on the intensity in the atypical or dysplastic area relative to background non dysplastic mucosa, or for non-dysplastic cases in the area of greatest intensity. This approach is described in detail in Kaye et al. ${ }^{3}$, and is similar to that used in Kaye et al. ${ }^{4}$ and Kastelein et al. ${ }^{5}$ The p53 staining was thus scored as negative (0), positive (1), absent (2), equivocal (3) and not representative (4). Positive expression and the "absent pattern" are regarded as reflecting abnormal p53 expression and likely p53 mutation ${ }^{4}$. The MDM2 nuclear staining was scored as absent (0), weak (1), moderate (2), strong (3) and not representative (4). The p53 staining was scored as negative (0), positive (1), absent (2), equivocal (3) and not representative (4). Similar to p53, this was assessed in atypical/dysplastic areas or in area of greatest intensity in non-dysplastic cases, and was based purely on intensity.

$62 \%$ of p53 positive cases had strong MDM2 staining versus $34 \%$ with negative p53 (fishers exact test: $p=0.002$ ). There was a positive correlation (Spearman's rho value $=0.47$, $p<0.001$ ) with increasing MDM2 staining as the grades of dysplasia increased (Table 1 ). However, there was no association (fishers exact test: $p=1$ ) between MDM2 staining intensity in ND/ID cases and progression to dysplasia or cancer (Table 2). Follow up times were however shorter in the MDM2 positive group (mean 15.5 months vs 36.5 months, $\mathrm{P}=0.04)$.

The positive association between abnormal p53 expression and MDM2 expression is counterintuitive since MDM2 is generally regarded as a negative regulator of $\mathrm{p} 53$. A previous study investigated the relationship between MDM2 and p53 mutation in OAC and BO specimens. This found, in line with established dogma, a negative association between TP53 mutation and MDM2 expression. However they did find, similar to our own study, that MDM2 expression tended to correlate with strength of $p 53$ staining. A possible reason for 
this discrepancy is that only part of the $\mathrm{p} 53$ gene was tested for mutations and it likely missed some mutations ${ }^{6}$.

Another study in OAC by quantitative PCR and FISH showed $6 \%$ of OAC cases had definite MDM2 gene amplification and concurrent enhanced immunohistochemical expression of MDM2 protein but with no correlation to p53 mutation ${ }^{7}$. As this study was in advanced $\mathrm{OAC}$, it is not known if this represented an early or late event in Barrett's carcinogenesis. The mechanism may be related to genomic doubling that has been recently proposed to be an important but late event in in the setting of $\mathrm{BO}^{8}$.

Our data may be explained by the theory that MDM2 can be stabilised by mutant p53. Peng et al. found that mutant $p 53$ resulted in the stabilisation and accumulation of MDM2 in a wide variety of epithelial and mesenchymal cancers ${ }^{9}$. Alternatively, our data may reflect the fact that MDM2 does have functions independent of p53; it may act in synergy with mutant p53 to drive dysplasia progression ${ }^{10}$.

Thus we have unequivocally shown that increased MDM2 expression occurs more frequently with higher grades of dysplasia, alongside abnormal p53 expression. However, MDM2 expression is not a useful marker for predicting progression to OAC. Future research should continue to investigate whether MDM2 biologically is relevant in the development of OAC.

\section{References}

1. Fitzgerald RC, di Pietro M, Ragunath K et al. British Society of Gastroenterology guidelines on the diagnosis and management of Barrett's oesophagus. Gut. 2014; 63; 7-42.

2. Wade M, Li Y-C, Wahl GM. MDM2, MDMX and p53 in oncogenesis and cancer therapy. Nat. Rev. Cancer. 2013; 13; 83- PubMed ;96.

3. Kaye PV, Ilyas M, Soomro I et al. Dysplasia in Barrett's oesophagus: p53 immunostaining is more reproducible than haematoxylin and eosin diagnosis and improves overall reliability, while grading is poorly reproducible. Histopathology. 2016; 69; 431-440

4. Kaye PV, Haider SA, James PD et al. Novel staining pattern of p53 in Barrett's dysplasia-the absent pattern. Histopathology. 2010; 57; 933-935 PubMed .

5. Kastelein F, Biermann K, Steyerberg EW et al. Aberrant p53 protein expression is associated with an increased risk of neoplastic progression in patients with Barrett's Oesophagus. Gut. 2013; 62; 1676-1683

6. Soslow RA, Altorki NK, Yang $\mathrm{G}$ et al. mdm-2 expression correlates with wild-type p53 status in esophageal adenocarcinoma. Mod. Pathol. 1999; 12; 580- PubMed ;586.

7. Michalk M, Meinrath J, Künstlinger $\mathrm{H}$ et al. MDM2 gene amplification in esophageal carcinoma. Oncology Reports. 2016; 35; 2223-2227

8. Stachler MD, Taylor-Weiner A, Peng S et al. Paired exome analysis of Barrett's oesophagus and adenocarcinoma. Nat Genet. 2015; 47; 1047-1055

9. Peng Y, Chen L, Li C et al. Stabilization of the MDM2 oncoprotein by mutant p53. J. Biol. Chem. 2001; 276; 6874- PubMed ;6878.

10. Ganguli G, Wasylyk B. p53-Independent Functions of MDM2. Mol. Cancer Res. 2003; 1; 1027-1035 PubMed 


\section{Acknowledgements}

Leora Robinson: Study design, performed benchwork, IHC interpretation, wrote paper Mohammad llyas: Edited Paper

Krish Ragunath: Study design

Tim Card: Study design

Berice Abradu-berchie: Performed benchwork, IHC interpretation

Philip Kaye: Conception, study design, pathology interpretation, edited paper

\section{Ethics}

Nottingham Health Science Biobank Board ACP 000035 


\section{Tables}

Table 1. Expression of MDM2 in the progression of BO

\begin{tabular}{l|lcccc|}
\multicolumn{1}{c}{$\begin{array}{l}\text { MDM2 } \\
\text { Intensity }\end{array}$} & \multicolumn{2}{l|}{} \\
\hline & 0 & 1 & 2 & 3 & Total \\
No dysplasia & 1 & 10 & 2 & 0 & 13 \\
Indefinite & 1 & 16 & 8 & 1 & 26 \\
LGD & 0 & 10 & 10 & 1 & 21 \\
HGD & 0 & 3 & 8 & 2 & 13 \\
Carcinoma & 1 & 0 & 5 & 3 & 9 \\
Total & 3 & 39 & 33 & 7 & 79
\end{tabular}

Spearman's rho value $=0.47, p<0.001$

Table 2 Progression of non dysplastic and indefinite for dysplasia BO in relation to MDM staining

\begin{tabular}{l|lll|}
\multicolumn{4}{c}{ MDM 2 } \\
\multicolumn{1}{c|}{ Intensity } & & \\
\hline Progressed & Weak & Strong & Total \\
No & 16 & 7 & 23 \\
Yes & 12 & 4 & 16 \\
& 28 & 11 & 39 \\
\cline { 2 - 4 } & &
\end{tabular}

Fishers exact test: $p=1$ 


\section{Figures}

Figure 1: Barrett's oesophagus, no dysplasia (A) H\&E; (B) p53 negative; (C) MDM2 score 1

Figure 2: Barrett's oesophagus, high-grade dysplasia (A) H\&E; (B) p53-absent pattern; (C) MDM2 dysplastic glands score 3. 